\title{
Characteristic imaging features of neurovascular involvement in primary Sneddon's syndrome: an analysis of 12 cases
}

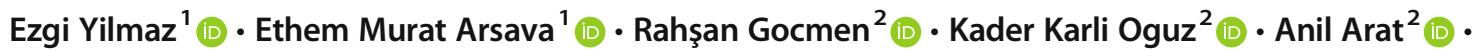 \\ Mehmet Akif Topcuoglu ${ }^{1}$
}

Received: 26 April 2020 / Accepted: 19 July 2020

(C) Fondazione Società Italiana di Neurologia 2020

\begin{abstract}
Objective Sneddon's syndrome is a cerebrocutaneous non-inflammatory progressive distal arteriopathy, characterized by livedo racemosa, stroke, and neuropsychiatric symptoms. Our aim was to highlight the characteristic neuroimaging features of Sneddon's syndrome that might be helpful to clinicians in timely diagnosis of this entity.

Methods Twelve patients (median age 49 years, 11 female) with primary Sneddon's syndrome, diagnosed in last 10 years, were analyzed from the perspective of magnetic resonance imaging (MRI) features. In addition, a novel pseudoangiomatosis score was defined for grading angiographic abnormalities (range: 0 to 6).

Results Median interval from the onset of neurological symptoms to diagnosis was 6 years. Presentation was with acute stroke in 5, seizures in 3, dementia/speech problems in 2, seizures plus cognitive dysfunction in 1, and chronic progressive hemiparesis in 1. All patients had a typical lesion pattern on MRI. This included multiple (median 3) cortical-subcortical supratentorial and cerebellar non-territorial infarcts, accompanied by multifocal cerebral atrophy. Of note, large territorial infarcts due to cerebral parent artery occlusion, an embolic pattern with multi-territorial involvement on diffusion-weighted imaging, small vessel disease features like severe white matter involvement or lacunar infarcts, and cerebral hemorrhage in the absence of anticoagulation were not observed. MRI lesion severity was not correlated with angiographic arteriopathy severity, clinical stage, or presentation symptoms.

Conclusion Sneddon's syndrome is characterized by highly typical clinico-radiological features. Brain MRI has diagnostic value. By knowing the characteristics of the syndrome, misdiagnosis and potentially harmful treatment can be prevented in this entity that might pose a diagnostic challenge.
\end{abstract}

Keywords Arteriopathy $\cdot$ Vasculopathy $\cdot$ Vasculitis $\cdot$ Cerebral embolism $\cdot$ Endocarditis $\cdot$ Hemorrhage

\section{Introduction}

Sneddon's syndrome is a progressive cerebrocutaneous noninflammatory distal arteriopathy. It was named after Dr. Ian

Electronic supplementary material The online version of this article (https://doi.org/10.1007/s10072-020-04621-0) contains supplementary material, which is available to authorized users.

Mehmet Akif Topcuoglu

mat@hacettepe.edu.tr; matopcuoglu@yahoo.com

Ezgi Yilmaz

igezgi@hotmail.com

Ethem Murat Arsava

arsavaem@hotmail.com

Rahşan Gocmen

gocmentr@yahoo.com
Bruce Sneddon, a British dermatologist who described the syndrome as a separate entity characterized by combination of cerebrovascular disease and livedo racemosa [1]. Its incidence is very rare, approximately 4 per million, and almost
Kader Karli Oguz

karlioguz@yahoo.com

Anil Arat

anilarat@hotmail.com

1 Neurology Department, Hacettepe University Hospitals, 06100, Sihhiye, Ankara, Turkey

2 Radiology Department, Hacettepe University Hospitals, 06100, Sihhiye, Ankara, Turkey 
completely affects women in their thirties to forties. It is usually sporadic, but families have been reported with autosomal dominant inheritance showing an incomplete penetrance pattern [2].

The clinical picture in Sneddon's syndrome is actually quite homogenous. Livedos precede cerebrovascular involvement by years, and dementia, if occurs, by decades. However, it is usually misdiagnosed as central nervous system (CNS) vasculitis, particularly primary CNS angiitis (PACNS), CNS vasculopathy, and antiphospholipid antibody syndrome (APLAS), probably due to unfamiliarity to the clinical, neuroimaging, and angiographic characteristics of this syndrome. Indeed, Sneddon's syndrome is often considered when standard treatments for PACNS fail [3].

We believe that Sneddon's syndrome can be largely diagnosed by its highly typical stroke phenomenology, if the characteristic magnetic resonance imaging (MRI) features are taken into account, thereby averting the administration of futile treatments that might be potentially harmful. To support this view, multimodal MRI features of "the syndrome," and the accompanying angiographic characteristics are herein revisited with addition of our newly defined findings that might potentially be of diagnostic value.

\section{Patients and methods}

\section{Patients}

Five patients with Sneddon's syndrome presenting with acute stroke were extracted from the prospectively gathered stroke database of our hospital, referring to approximately $0.1 \%$ of acute ischemic stroke patients hospitalized in the last 10 years. By combining this cohort, with 4 patients who are being followed in our outpatient clinic and 3 additional patients referred to our center in the same period, a total of 12 patients with diagnosis of Sneddon's syndrome were included into this analysis.

\section{Diagnosis}

The diagnosis of primary Sneddon's syndrome was established on the basis of recurrent/progressive ischemic neurovascular syndrome and typical generalized livedo rasemosa, confirmed by dermatologist examination and absence of any other disease or condition that may cause the relevant symptoms and signs $[2,4]$. Patients with positive clinical and blood markers for specific hematological conditions such as hereditary thrombophilia, autoimmune syndromes such as APLAS, and rheumatologic diseases such as systemic lupus erythematosus (SLE) were excluded.

The course of the disease was categorized into three phases according to the degree of neurological involvement: Phase 1 constituted the "neuro-prodromal phase" characterized by nonspecific symptoms such as headache and dizziness. Phase 2 signified the "stroke phase," where recurrent and multifocal strokes and/or TIAs complicated the clinical course. Lastly, phase 3, the "neuropsychiatric phase," included features such as concentration deficit, inattention, disturbed visual perception, and visuospatial skills along with cognitive decline that culminated into "early-onset vascular dementia" at the final stages [5].

\section{Data}

\section{Demographic, clinical and laboratory}

For all patients, data regarding demographics (age, gender, age at diagnosis, interval between symptom onset and diagnosis, overall disease duration), neurological features (stroke location and symptoms, cognitive symptoms, mini-mental status examination-MMSE scores), systemic features (history of spontaneous abortion, cardiac evaluation including echocardiography and Holter monitoring), laboratory findings (cerebrospinal fluid-CSF analysis, serological markers for autoimmune diseases, detailed acquired and genetic thrombophilia panels), and treatment strategies were collected.

\section{Magnetic resonance imaging}

A total of 29 MRI studies were performed in our cohort of 12 patients: One in 4, two in 4, three in 2 , four in 1 , and seven in 1. We evaluated MRI at the time of diagnosis, and almost invariably closest to the time of catheter angiography, but we can also say that there was no significant pattern change on follow-up MRIs. MRIs were evaluated in terms of the presence of acute, subacute, and chronic infarction(s), cerebral microbleeds (CMBs), lacunes, leukoaraiosis, perivascular space, and subcortical and cortical atrophy [6].

Acute infarcts were diagnosed in the presence of territorial areas of restricted diffusion. Subacute infarcts were diagnosed in the presence of T2-shine through appearance. T2 bright lesions without any signal on diffusion-weighted images were classified as chronic infarcts. The number and localization of acute and chronic infarcts were determined. CMBs were defined as small-sized (maximum diameter less than $10 \mathrm{~mm}$ ) signal void areas visible on MR sequences sensitive to susceptibility effect such as $\mathrm{T} 2 *$ or susceptibility-weighted imaging (SWI) [6, 7]. Lacunes were subcortical CSF-filled round/ ovoid shape cavitations in the territories of perforating arteries, with diameters ranging from 3 to $15 \mathrm{~mm}$, and having a peripheral T2 hyperintense rim on fluid-attenuated inversion recovery [FLAIR] sequences. Noncavitating lacunes were not included into the analyses [6]. Leukoaraiosis (LA) term was preferred for defining the subcortical white matter hyperintensity lesions on FLAIR or T2-weighted sequences. Fazekas' scale was used to determine the extent and location 
of leukoaraiosis, which was categorized into "periventricular" leukoaraiosis (" 0 ": absent; " 1 ": caps or pencil-thin lining; " 2 ": smooth halo; and " 3 ": confluent hyperintensity extending into centrum semiovale) and "subcortical" ("deep" in original) leukoaraiosis (" 0 ": absent; " 1 ": punctate; " 2 ": non-confluent; and " 3 ": large coalescing hyperintensities) lesions [8]. Perivascular space comprised CSF-isointense, small (maximum cross-sectional diameter less than $3 \mathrm{~mm}$ ), linear, or round-shaped areas surrounding the perforating vessels, mainly veins. Enlarged perivascular spaces were graded (" 0 " for absence perivascular space; " 1 " mild 1-10; " 2 " moderate 1120; " 3 " frequent 21-40; and " 4 " severe more than 40) for basal ganglia, and subcortical white matter separately. The smaller size and normal appearance of adjacent white matter enabled the discrimination of perivascular spaces from lacunes [6]. Cerebral atrophy was graded both for "deep" referring ventricular enlargement and "superficial" referring sulcal enlargement using a modified 3-point scale as "absent," "mild," and "moderate to severe." This score was adapted from Farrel et al. [9].

\section{Angiography}

Four vessel angiogram finding were evaluated in terms of presence, severity, and extent of the distal (third order) arteriopathy. Distal arteriopathy was herein called as "pseudoangiomatosis." Pseudoangiomatosis includes mainly peripheral "pruning" of the distal cortical arteries along with irregularity, tortuosity, elongation, angulation, and microcoiling of corticomedullary branches and collaterals. Of note, the first and second order cerebral arterial segments were in normal shape and caliper in our patients. A pseudoangiomatosis score was described by addition of the grades ("grade-0" (absent), "grade-1" (minor, present in less than half of the territory), and "grade-2" (present in more than half of the territory)) evaluated separately in injections of the right and left carotid, and posterior circulation. The total pseudoangiomatosis score therefore ranged from 0 to 6 (Fig. 1).
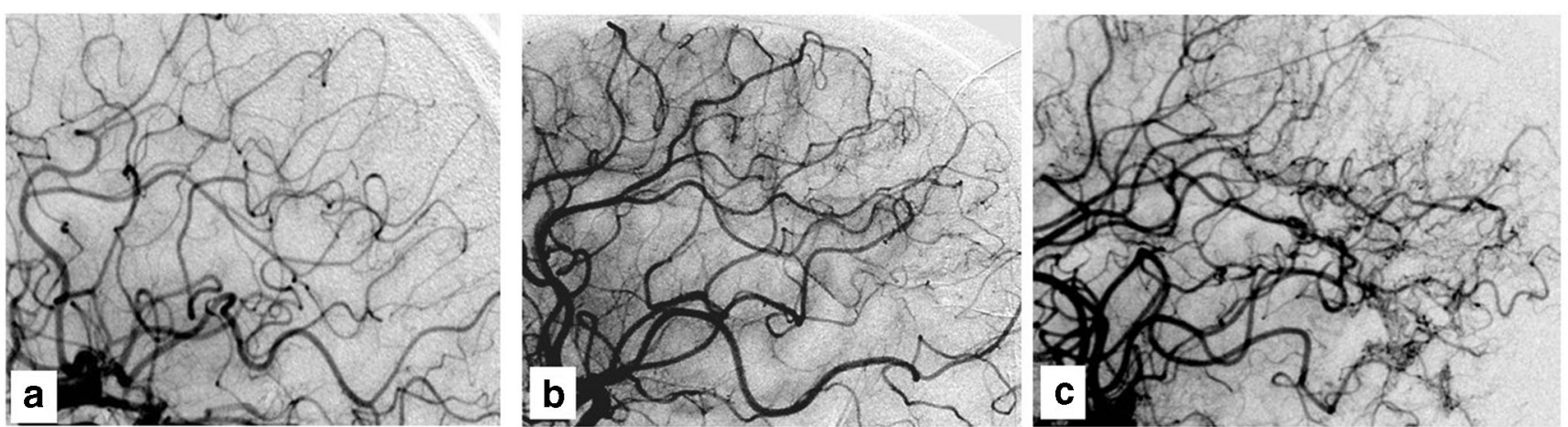

Fig. 1 Examples of pseudoangiomatosis grading: a Grade 0: Normal; b Grade-1, pseudoangiomatosis present, but less than half of the territory; c Grade2, pseudoangiomatosis evident in more than half of the territory

\section{Results}

Detailed individual clinical and imaging data of patients are shown at supplementary Tables 1 and 2. In brief, eleven of the 12 patients included were women. The median age was 49 (range, 35 to 53) at the time of enrollment, and 43 (range, 39 to 53) at the disease diagnosis. Median interval from the onset of neurological symptoms to our diagnosis was 6 years (range, 1 to 18), while the median of predicted disease duration was 9.5 years (range, 1 to 19 years).

\section{Clinical}

Three patients were categorized as phase 2 and the remaining as phase 3 disease. There were no phase 1 patients. None of the patients had a positive family history for a similar disease, extracerebral vascular event, or malignancy. Hypertension was detected in 5 patients, and diabetes mellitus in 1 patient. Two patients were active smokers, and two women had a spontaneous abortus history. Five patients presented with acute stroke. Other presentation modes were dementia and/ or speech problem in 2 , seizures in 3 , seizures plus cognitive dysfunction in 1, and chronic progressive hemiparesis in 1. Two of these seven did not, while five had remote, have overt stoke/TIA history.

All patients used antiplatelet (in combination in 7) or anticoagulant medications; specifically these included aspirin in 3 , aspirin plus dipyridamole in 4, aspirin plus clopidogrel in 2 , clopidogrel only in 1 , aspirin plus dipyridamole plus clopidogrel in 1, and warfarin in another. Immunosuppressive treatments were given to 3 patients (steroid plus cyclophosphamide in 2 and steroid in 1) with a presumptive diagnosis of PACNS.

\section{Laboratory}

Complete blood count, hepatic and renal function tests, lipid profile including lipoprotein (a) levels, $\mathrm{HbAlc}$, and urine 
analysis were normal. Blood biomarkers related to infections, including erythrocyte sedimentation rate, CRP levels, HIV, and viral hepatitis serology, were also within normal limits in all patients.

Repeated testing for antiphospholipid syndromes by assays such as serum anticardiolipin and beta-2glycoprotein-I antibodies and lupus anticoagulant remained within normal level or negative. Antinuclear antibodies were positive in 8 patients; the titers were $1 / 100$ in $6,1 / 160$ in 1 , and $1 / 320$ in another. No abnormality was evident for other serological markers of systemic vasculitis. Hereditary thrombophilia screen was negative in 4 , but disclosed methylene tetrahydrofolate reductase C677T homozygous mutation in 2 and heterozygous mutations in another 2; methylene tetrahydrofolate reductase A1298C homozygous mutations in 2; plasminogen activator inhibitor-1 4G/5G heterozygous polymorphism in 3; and factor V Leiden heterozygosity in 1 (three patients had two concomitant abnormalities). Homocysteine concentrations were mildly elevated in 5 patients, and no other history that might be suggestive of thrombophilia like deep vein thrombosis or pulmonary embolism was present in any of these patients.

CSF examination was performed in 8 patients, and was normal in terms of glucose, protein, cell count, immunoglobulin-G index, and oligoclonal bands.

Transthoracic echocardiography and Holter monitoring, performed in 11 and 10 patients, respectively, were normal. Of note, no significant heart valve abnormalities were observed; two patients had mild mitral valve insufficiency, four had mitral valve thickening to some extent, and one had septal hypokinesis on echocardiography. No patient had paroxysmal atrial fibrillation.

\section{MRI}

MRI documented (sub) acute infarction in six. These were cortical-subcortical small- to medium-sized middle cerebral artery (MCA) branch infarcts in 2, small MCA cortical infarcts in 3 (asymptomatic in 1), and territorial large posterior cerebral artery (PCA) infarction in another. All patients had at least one cortical/subcortical medium-sized chronic infarct (Fig. 2). Median infarct number was 3 (range, 1 to 5); 1 in three, 2 in one, 3 in three, 4 in three, and 2 in five patients. Subcortical small-sized old infarctions are unusual, and present in only one patient (case 3, Fig. 2). All cortical-subcortical infarcts were located in supratentorial regions; on the other hand, all patients had at least one cerebellar non-territorial infarct (multiple in 9, one in 2, and minor in 1, Fig. 3).

CMBs were detected in a particular patient, who had 10 CMBs consistent with a hypertensive pattern, and absent in 9 . Only a single patient had one lacunar infarct. No significant leukoaraiosis, or white matter hyperintensities, was detected in these patients. Fazekas' periventricular grade was 1 in all except for one (0), and Fazekas' deep white matter hyperintensity score was given as zero in 5 and 1 in seven. Basal ganglia perivascular spaces were usually in normal range as well, and scored 1 in all. Subcortical perivascular spaces were detected in 10 patients, and its burden was graded as 1 in five, 2 in three, and 3 in two patients. Deep cerebral atrophy was present in 11 ; most were rated as grade-1 $(n=8$; two phase 2 and six phase 3 patients). The atrophy was considered significant (i.e., grade-2) in the remaining three patients, all of whom were phase 3. Superficial atrophy was noted for all patients regardless of the disease phase: grade-1 for 3 phase 2 patients and 7 phase 3 patients. Severe atrophy was noted in two phase 3 patients.

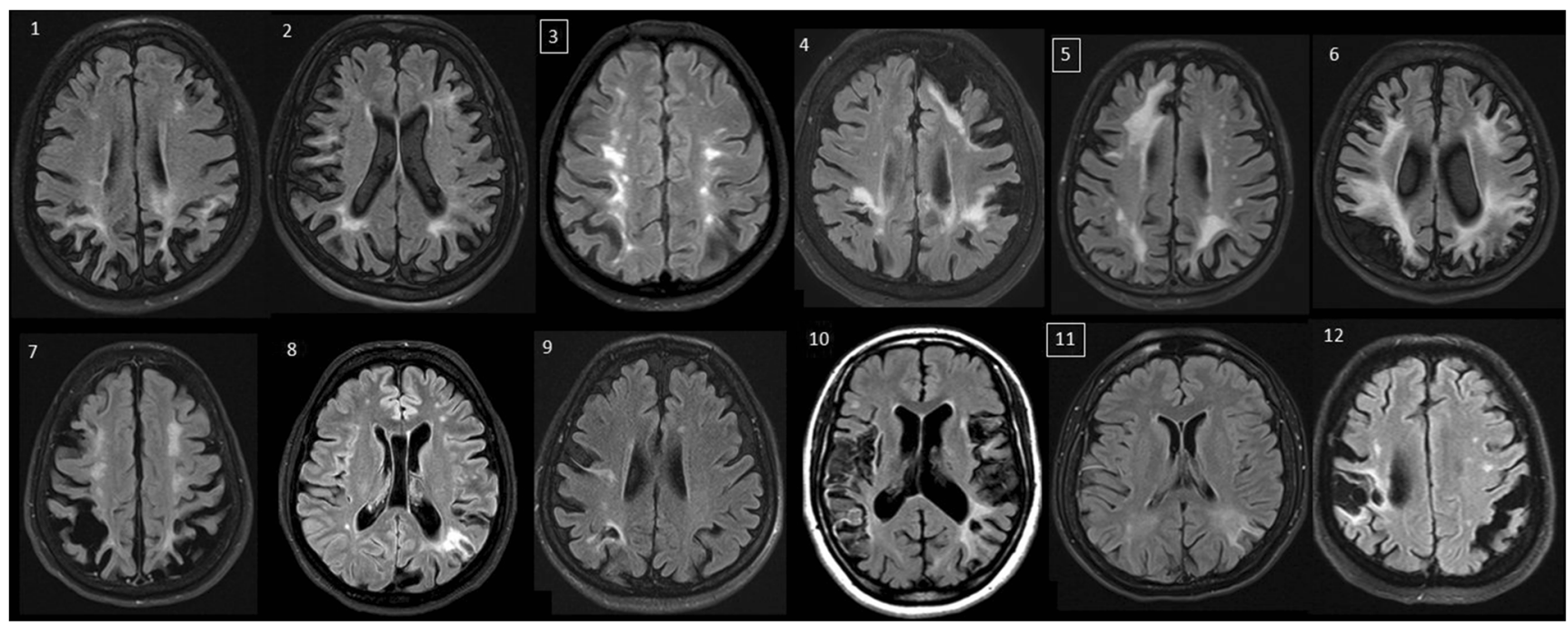

Fig. 2 Representative supratentorial MRI sections of all patients are shown. The numbers are in the patient order. Boxed ones are the numbers of patients categorized as phase 2. Detailed clinical, laboratory, and imaging findings of the patients are given in Supplementary web Table 1 and 2 


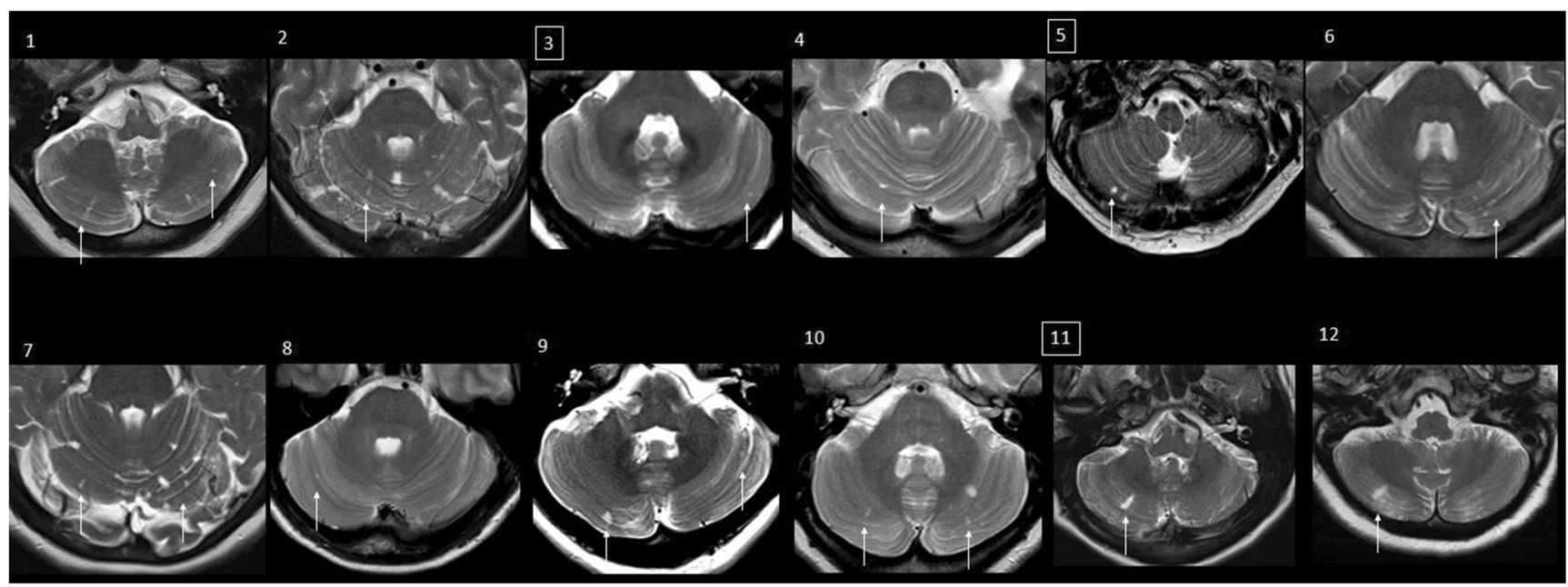

Fig. 3 Representative infratentorial MRI sections of all patients are shown. The numbers are in the patient order. Boxed ones are the numbers of patients categorized as phase 2. Detailed clinical, laboratory, and imaging findings of the patients are given in Supplementary web Table 1 and 2

\section{Cerebral angiography}

Catheter angiography was performed in 9 patients and documented pseudoangiomatosis pattern in all. In patients categorized into phase 2 disease, the severity was slightly less than the others (Supplementary Table 2). In 2 patients with phase 2 syndrome, the total score was 1 and 4 . In contrast, median of score in other patients was 5 . In 7 patients with phase 3 disease and angiography, total pseudoangiomatosis score was 6 in 4 and 5 in 3 patients. All phase 3 patients had significant supratentorial arteriopathy, always more severe than the posterior circulation.

\section{Discussion}

In Sneddon's syndrome, it is known that cortical infarcts due to distal cerebral arteriopathy accumulate over time, and the addition of numerous large and small cortical infarcts causes significant cerebral atrophy and neuropsychiatric symptoms $[2,3,5,10]$. An example of this dramatic situation is given in Fig. 4. This type of covert infarct accumulation is not a presentation mode of other neurovascular syndromes. Based on imaging topography and morphology, Bottin et al. classified Sneddon-associated cerebral infarctions into three groups as large territorial cortical-subcortical, distal superficial

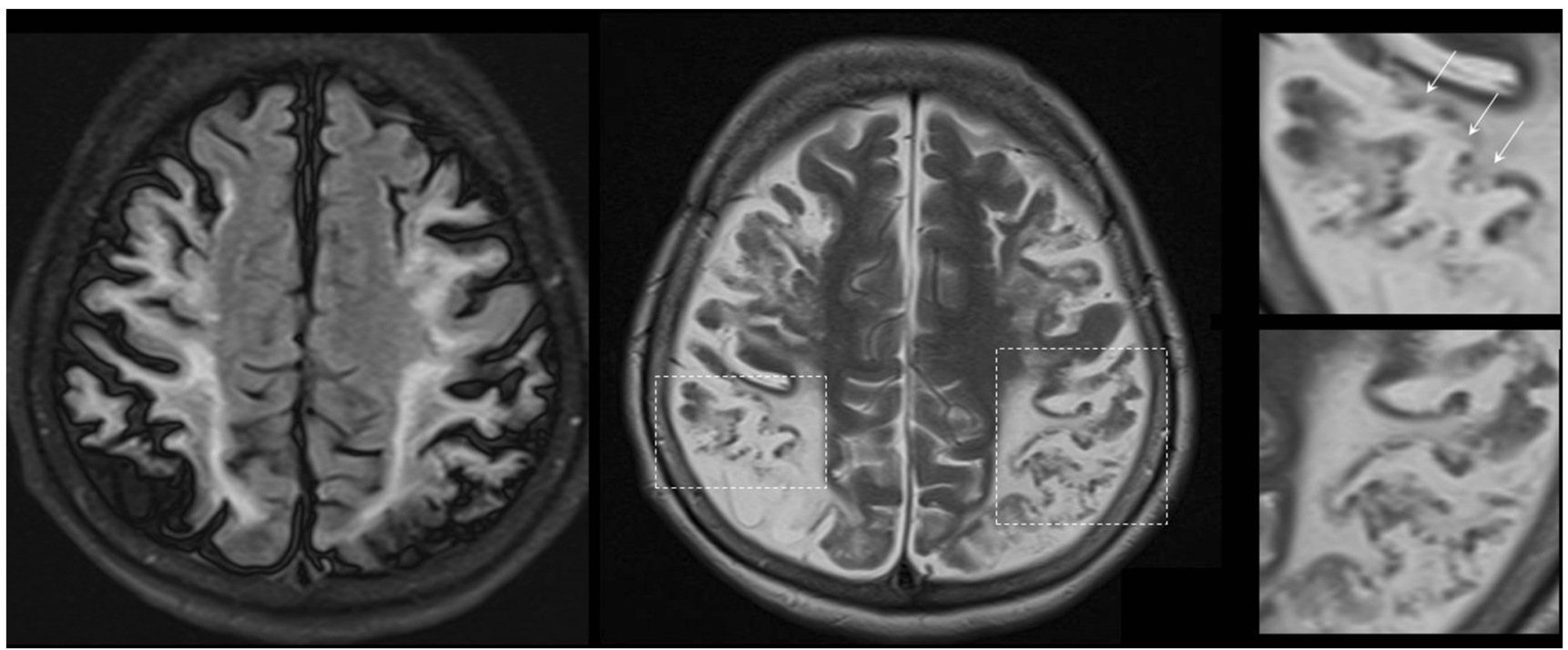

Fig. 4 Example of advanced neuro Sneddon disease is herein documented. Typical images of multifocal cerebral atrophy due to countless small cortical infarctions are seen in FLAIR and T2-weighted images. The boxes were enlarged to discern cortical infarcts better (sampled with arrows) 
cortical, and deep white matter infarcts (similar to hypertensive lacune) [4]. In our series, we found that lacunar infarctions are very rare, which may be related to the fact that our patients were mostly normotensive. On the other hand, we did not have hemorrhage, and this was due to our strategy of avoiding anticoagulants, in the absence of APLAS [3]. In addition, exclusion of significant cardioembolic etiologies has allowed us to delineate the specific arteriopathic syndrome caused by Sneddon's syndrome in our series. Therefore, our series are quite homogeneous in this respect. Our experience indicates that effective hypertension control and avoidance of liberal anticoagulants are keys to prevent intracerebral bleeding associated with angiomatosis in Sneddon syndrome.

We have documented herein that Sneddon's arteriopathy leads to a very typical and recognizable neuroimaging pattern. This observation is not novel, as seen by the images available in the literature showing lesions with similar characteristics; however, we wanted to emphasize the importance of this pattern once more in this descriptive analysis. The typical appearance includes multiple cortical-subcortical medium to smallsized infarcts and cerebellar non-territorial infarct(s) in the background of mild cerebral diffuse plus focal atrophy. The presence of these findings altogether in a young woman without significant risk factors should always lead us to the diagnosis of Sneddon's syndrome. Of note, a low-titer ANA can be detected in more than half of the patients, and is compatible with this syndrome. If livedo is detected, then the diagnosis is established. There are cases in which livedo is diagnosed after a stroke in the germane literature $[11,12]$. This highly uniform appearance gives us the idea that MRI can be considered a biomarker of this disease.

In our opinion, catheter angiography does not seem necessary for the diagnosis of Sneddon's syndrome, but if performed, it has distinctive findings as well, and is therefore grossly diagnostic. Typically the first and second order cerebral arterial tree is completely normal, and the arteriopathy is confined to the third order vessels in this syndrome. There is no direct correlation between the grade of this angiographic involvement, like degree of MRI-detected atrophy and clinical severity, as we confirmed here. In addition, the high prevalence of silent cortical infarcts is remarkable. These points all seem to be suggestive of this disease.

In addition to the rather typical lesion pattern, the following findings are also probably specific for Sneddon's syndrome: First, the diffusion-weighted sequence in Sneddon's syndrome patients never shows embolic patterns such as multi-territorial multiple infarcts. Second, white matter involvement is always very mild. Basal arteries and perforators are not principally involved in this disease. Thirdly, although diffuse cerebral atrophy is generally mild, significant focal tissue loss as a sequela of covert infarctions that usually superimpose and lead to disabling neurological dysfunction and epilepsy.
Epilepsy is considered to develop due to silent cortical infarctions and may be the initial manifestation of the disease. Fourthly, no large territorial infarcts are seen in this disease. A large territorial PCA infarction was noted in one of our patients that might be suggestive of embolism. However, this patient had also typical former lesions. As an additional feature, we have never seen a hemorrhagic stroke because most of our patients have not received anticoagulants. Especially if there is an advanced pseudoangiomatosis pattern on angiography, the risk of bleeding may be high. And also, we have never seen cortical-subcortical infarcts in infratentorial regions. $\mathrm{We}$, indeed, failed to find such pictures in the literature, suggesting that this should be a rarity; albeit some publications said it may be up to $15 \%$ [4]. Last, perhaps the most important, cerebellar non-territorial infarcts can be typical for this syndrome. Their existence was mentioned in some of the previous publications [4], but its originality has not been emphasized. The presence of non-territorial dot- or crayon-like cerebellar infarcts, which was invariably evident in our patients (Fig. 3), is reasonable because it has previously been shown that an underlying hypercoagulable state is significantly higher in such lesions [13].

In conclusion, Sneddon's syndrome is a neurological rarity, but it is very often included in the differential diagnosis of other neurological diseases. Therefore, it is critical to recognize it in detail. In this article, we state that brain MRI findings are quite typical and might be diagnostic, even if the clinical picture and the presence of livedo racemosa, or perhaps both, are unknown. The presence of multiple cortical infarcts along with crayon-shaped nonterritorial cerebellar infarcts in a young woman should definitely suggest Sneddon's syndrome. We even think that skin biopsy is not necessary for diagnosis. Biopsy may be important in Sneddon's Syndrome presenting only with dermatological findings. In other cases, neuro Sneddon can be easily recognized by this MRI appearance.

We herein shared our experience with primary Sneddon syndrome. The number of cases in our study is not sufficient to make definitive generalizations, although the syndrome is quite rare. Participation of a single center is also a limitation. Multicenter and prospective studies of this disease are required to crystallize our observations further.

Availability of data and material If there is a need more than supplementary data already provided, all will be provided upon reasonable request.

\section{Compliance with ethical standards}

Conflict of interest The authors declare that they have no conflict of interest.

Ethical approval Waived for this analysis. Stroke database and its protocols were approved by university ethical committee. 


\section{References}

1. Sneddon IB (1965) Cerebrovascular lesions and livedo reticularis. Br J Dermatol 77:180-185

2. Wu S, Xu Z, Liang H (2014) Sneddon's syndrome: a comprehensive review of the literature. Orphanet J Rare Dis 9:215. https://doi. org/10.1186/s13023-014-0215-4

3. Samanta D, Cobb S, Arya K (2019) Sneddon syndrome: a comprehensive overview. J Stroke Cerebrovasc Dis 28(8):2098-2108. https://doi.org/10.1016/j.jstrokecerebrovasdis.2019.05.013

4. Bottin L, Frances C, de Zuttere D, Boelle PY, Muresan IP, Alamowitch S (2015) Strokes in Sneddon syndrome without antiphospholipid antibodies. Ann Neurol 77(5):817-829. https:// doi.org/10.1002/ana.24382

5. Zelger B, Sepp N, Stockhammer G, Dosch E, Hilty E, Ofner D, Aichner F, Fritsch PO (1993) Sneddon's syndrome. A long-term follow-up of 21 patients. Arch Dermatol 129(4):437-447. https:// doi.org/10.1001/archderm.129.4.437

6. Wardlaw JM, Smith EE, Biessels GJ, Cordonnier C, Fazekas F, Frayne R, Lindley RI, O'Brien JT, Barkhof F, Benavente OR, Black SE, Brayne C, Breteler M, Chabriat H, Decarli C, de Leeuw FE, Doubal F, Duering M, Fox NC, Greenberg S, Hachinski V, Kilimann I, Mok V, Oostenbrugge R, Pantoni L, Speck O, Stephan BC, Teipel S, Viswanathan A, Werring D, Chen C, Smith C, van Buchem M, Norrving B, Gorelick PB, Dichgans M, nEuroimaging STfRVco (2013) Neuroimaging standards for research into small vessel disease and its contribution to ageing and neurodegeneration. Lancet Neurol 12(8):822-838. https://doi.org/10.1016/S1474-4422(13)70124-8

7. Buyukserbetci G, Saka E, Oguz KK, Gocmen R, Arsava EM, Topcuoglu MA (2018) Cognitive dysfunction in relation to topography and burden of cerebral microbleeds. Noro Psikiyatr Ars 55(1):84-90. https://doi.org/10.29399/npa.23018

8. Fazekas F, Chawluk JB, Alavi A, Hurtig HI, Zimmerman RA (1987) MR signal abnormalities at $1.5 \mathrm{~T}$ in Alzheimer's dementia and normal aging. AJR Am J Roentgenol 149(2):351-356. https:// doi.org/10.2214/ajr.149.2.351

9. Farrell C, Chappell F, Armitage PA, Keston P, Maclullich A, Shenkin S, Wardlaw JM (2009) Development and initial testing of normal reference MR images for the brain at ages 65-70 and 75-80 years. Eur Radiol 19(1):177-183. https://doi.org/10.1007/ s00330-008-1119-2

10. Marinho JL, Piovesan EJ, Leite Neto MP, Kotze LR, Noronha L, Twardowschy CA, Lange MC, Scola RH, Zetola VH, Novak EM, Werneck LC (2007) Clinical, neurovascular and neuropathological features in Sneddon's syndrome. Arq Neuropsiquiatr 65(2B):390 395. https://doi.org/10.1590/s0004-282x2007000300005

11. Cavestro C, Richetta L, Pedemonte E, Asteggiano G (2009) Sneddon's syndrome presenting with severe disabling bilateral headache. J Headache Pain 10(3):211-213. https://doi.org/10. 1007/s10194-009-0109-3

12. Killeen T, Wanke I, Mangiardi J, Cesnulis E (2014) Ruptured, fusiform, distal lenticulostriate aneurysm causing intraventricular haemorrhage in a patient with antiphospholipid-negative Sneddon's syndrome. Clin Neurol Neurosurg 116:80-82. https:// doi.org/10.1016/j.clineuro.2013.11.009

13. Amarenco P, Levy C, Cohen A, Touboul PJ, Roullet E, Bousser MG (1994) Causes and mechanisms of territorial and nonterritorial cerebellar infarcts in 115 consecutive patients. Stroke 25(1):105112. https://doi.org/10.1161/01.str.25.1.105

Publisher's note Springer Nature remains neutral with regard to jurisdictional claims in published maps and institutional affiliations. 